\title{
Statistical Treatment of Activity and Durability of Electrocatalysts with Distributed Binding Energies
}

\author{
Jun Huang ${ }^{1}$ \\ Institute of Energy and Climate Research: Theory and Computation of Energy Materials (IEK-13), \\ Forschungszentrum Jülich GmbH, Jülich 52425, Germany \\ Email address: j.huang@fz-juelich.de
}

\begin{abstract}
We present a statistical treatment of the catalytic activity and durability of nonhomogeneous electrocatalysts that possess distributed binding energies of reaction intermediates. The treatment is simple, generic, and amenable to analytical solutions. It is revealed that the highest overall catalytic activity is obtained with a suitable level of nonhomogeneity that is commensurate with the average property. The evolution of the binding energy distribution is described by the Fokker-Planck theory. Exponential decay of the catalytic activity is predicted theoretically and confirmed experimentally. The exponential decay shows one- or two stages, depending on the initial distribution properties. The present work represents a step toward closing the gap between ideal and practical electrocatalysts using statistical considerations.
\end{abstract}

1 Present address: Institute of Theoretical Chemistry, UIm University, 89069 Ulm Germany 


\section{Introduction}

An electrocatalytic reaction, for example, the oxygen reduction reaction (ORR) in polymer electrolyte fuel cells, proceeds via a complex reaction network consisting of several adsorbing intermediates $\left(\mathrm{OH}_{\mathrm{ad}}, \mathrm{O}_{\mathrm{ad}}, \mathrm{OOH}_{\mathrm{ad}}\right)$ and many elementary steps. Interlinking the catalytic activity with binding energies of adsorbing intermediates, denoted by $\Delta \mathrm{G}_{\mathrm{i}}$ for adsorbate $i$, is a major straddle in comprehending and controlling the catalyst dependence of catalytic activity.[1-4] Furthermore, binding energies of different intermediates are found to linearly correlate with each other. $[5,6]$ For the case of ORR, scaling relations, $\Delta \mathrm{G}_{\mathrm{OOH}}=\Delta \mathrm{G}_{\mathrm{OH}}+3.2 \mathrm{eV}$, and $\Delta \mathrm{G}_{\mathrm{O}}=$ $2 \Delta \mathrm{G}_{\mathrm{OH}}$, have been found. $[7,8]$ Consequently, one of the binding energies, say $\Delta \mathrm{G}_{\mathrm{OH}}$ for the ORR, can be chosen as the descriptor of a class of catalysts for a particular electrocatalytic reaction. This theory-guided approach of catalyst design is termed the binding-energy approach.

The relation between the catalytic activity and the chosen descriptor usually manifests in a volcano plot. The catalytic activity increases until a maximum is reached, and then declines as the descriptor increases further. The basic idea refers to the well-known Sabatier principle[9], indicating that the optimum catalyst binds the reaction intermediates neither too strongly nor too weakly.[10, 11] This binding-energy approach prevails, with proven efficacy, in electrocatalysis.[12, 13]

In many applications, such as fuel cells and water splitting devices, catalysts are usually present in the form of nanoparticles because of their increased surface area. In contrast to ideal catalysts, e. g., atomically well-defined single crystals,[14-16] practical catalysts have a large variety of active sites at the atomic level.[17, 18] These active sites reveal different catalytic activity as demonstrated in high-resolution catalytic activity mapping studies.[19-24] Consequently, it is important to understand the influence of nonhomogeneities on the overall catalytic activity and durability.[25]

Following the binding-energy approach, it appears straightforward to endow the chosen descriptor with a probability distribution function (PDF) to represent, as a first approximation, the heterogeneity among active sites. So far, only a few works have addressed such statistical issues in electrocatalysis. Norskov et al. presented a conceptual analysis on the varying 
contributions of different types of active sites to the overall catalytic activity.[10] Eikerling et al. modelled the degradation of the electrochemical surface area of Pt-based ORR catalysts using a statistical treatment of the growth of Pt nanoparticles.[26-28] Inspired by these works, herein, we present a statistical treatment to interlink the overall catalytic activity and durability of nonhomogeneous catalysts that have spatially distributed and temporally evolving binding energy with the PDF. The treatment may spur further progress in understanding heterogeneity in electrocatalysis.

\section{Method}

We make following assumptions, for the sake of obtaining analytical solutions, in this work. First, the heterogeneity is reflected solely by a distribution in the activity descriptor, namely, the binding energy of a certain reaction intermediate. It has become clear how the binding energy is determined by electronic factors of the catalyst, $[1,13]$ and the local electric field in the solution phase.[29, 30] The first assumption relieves us of treating multistep kinetics of the complex reaction network, which was handled using microkinetic modelling in our previous works[31-33] and others'.[34-36] This assumption is essentially the potential-determining step (PDS) approximation, whose limitations have been articulated by Koper.[37]

Second, the descriptor's distribution is characterized by a normal distribution

$$
p(x)=\frac{1}{\sqrt{2 \pi} \sigma} \exp \left(-\frac{1}{2}\left(\frac{x-\mu}{\sigma}\right)^{2}\right),
$$

where $x(\mathrm{eV})$ is the activity descriptor, $\mu(\mathrm{eV})$ the mean value, and $\sigma(\mathrm{eV})$ the standard deviation. Third, we assume that the catalytic activity is a symmetric volcano-shaped function of $x$. After normalization with respect to the maximum activity, the volcano plot is expressed as

$$
f(x)=\exp (-\beta|x|)
$$

where the coefficient is $\beta=\left(2 k_{B} T\right)^{-1}$ with $k_{B}\left(\mathrm{eV} \mathrm{K}^{-1}\right)$ being the Boltzmann's constant and $T(\mathrm{~K})$ the absolute temperature. At $T=300 \mathrm{~K}$, we have $\beta=19.4 \mathrm{eV}^{-1}$. 
The fraction of active sites falling into the descriptor range of $[x, x+d x]$ is $p(x) d x$, each contributing to the overall catalytic activity with an amount of $f(x)$. The overall catalytic activity is the sum of all active sites

$$
\langle f\rangle=\int_{-\infty}^{\infty} d x f(x) p(x)
$$

Some algebraic manipulations result in an analytical solution for $\langle f\rangle$

$$
\langle f\rangle=\frac{1}{2}\left(\exp \left(\frac{\beta^{2} \sigma^{2}+2 \beta \mu}{2}\right) \operatorname{erfc}\left(\frac{\beta \sigma^{2}+\mu}{\sqrt{2} \sigma}\right)+\exp \left(\frac{\beta^{2} \sigma^{2}-2 \beta \mu}{2}\right) \operatorname{erfc}\left(\frac{\beta \sigma^{2}-\mu}{\sqrt{2} \sigma}\right)\right),
$$

where $\operatorname{erfc}(x)$ is the complementary error function.

An electrocatalyst may degrade due to a plethora of mechanisms, such as, active site poisoning, support corrosion, dissolution and agglomeration of active sites, etc.[38-40] A comprehensive treatment of the degradation is beyond the scope of this work. Instead, we continue, in our line of reasoning, to interlink the degradation of the catalytic activity with temporal evolution of the binding energy distribution.

The fourth assumption says that the temporal evolution of the binding energy distribution follows a Markovian stochastic process, described by the Fokker-Planck theory [41]

$$
\frac{\partial p(x, t)}{\partial t}=\frac{\partial}{\partial x}\left(-\gamma(x, t) p(x, t)+D(x, t) \frac{\partial p(x, t)}{\partial x}\right)
$$

where the first term on the right hand side is a drift flux, and the second term is a diffusion flux. Generally, the drift coefficient $\gamma(x, t)$ and the diffusion coefficient $D(x, t)$ are time-varying and dependent on $x$.

According to the first assumption, the initial distribution is a normal distribution

$$
p(x, 0)=\frac{1}{\sqrt{2 \pi} \sigma_{0}} \exp \left(-\frac{\left(x-\mu_{0}\right)^{2}}{2 \sigma_{0}^{2}}\right),
$$

with $\mu_{0}$ and $\sigma_{0}$ being the initial mean value and standard deviation. A zero probability at infinity serves as the other boundary condition closing Eq.(5), 


$$
p( \pm \infty, t)=0 .
$$

Using constant $\gamma$ and $D$, we obtain

$$
p(x, t)=\frac{1}{\sqrt{2 \pi} \sigma(t)} \exp \left(-\frac{(x-\mu(t))^{2}}{2 \sigma(t)^{2}}\right),
$$

with a time-varying mean value

$$
\mu(t)=\mu_{0} \exp (\gamma t),
$$

and a time-varying standard deviation

$$
\sigma(t)=\sqrt{\frac{D(\exp (2 \gamma t)-1)}{\gamma}+\sigma_{0}^{2} \exp (2 \gamma t)} .
$$

As $p(x, t)$ retains a normal distribution along aging, the overall catalytic activity $\langle f\rangle$ is still expressed in the form of Eq.(4).

With the named assumptions, some of which are rather restrictive, the present treatment is helpless on simulating a practical catalyst without dramatic modifications of $p(x)$ and $f(x)$. That said, we hope the reader would agree that the treatment is not void of value as it is general to other forms of $p(x)$ and $f(x)$, for example, asymmetric volcano-shaped functions. In addition, we have known that volcano plots are potential-dependent;[42-45] it is thus important to note that the present treatment describes the performance of catalysts at constant potential, rather than constant current (density). This specification of the operating condition will be invoked in the selection of literature data to validate (more precisely, compare) the model with experiments.

\section{Results and discussion}

\section{Overall catalytic activity}

Figure 1 (a) depicts the variation of $\langle f\rangle$ as a function of $\sigma$ for different values of $\mu$, calculated using Eq.(4). Since $\langle f\rangle$ is an even function of $\mu$, we only consider positive values of $\mu$, corresponding to the weak-binding flank of the volcano plot. The analysis applies equally to the strong-binding flank of the volcano, i. e., $\mu<0$. When $\mu=0$ (the mean binding energy is 
reconciled with the volcano's apex), $\langle f\rangle$ monotonically decreases as $\sigma$ grows, implying that the overall catalytic activity of an averagely optimal electrocatalyst decreases rapidly when the homogeneity deteriorates. When $\mu \neq 0,\langle f\rangle$ changes nonmonotonically with increasing $\sigma$, implying that nonhomogeneity is not always detrimental. Specifically, $\langle f\rangle$ increases when $\sigma$ reaches $\sim \mu$, and then decreases slowly. An intuitive explanation of this peculiar nonmonotonicity is that a certain degree of nonhomogeneity, commensurate with the mean property, renders the highest fraction of "active sites" (no need to be specified in the current context) near the volcano's apex. In other words, for the vast majority of electrocatalysts whose mean property deviates from the optimum, the overall catalytic activity is highest when $\sigma \approx \mu$ instead of $\sigma=0$.
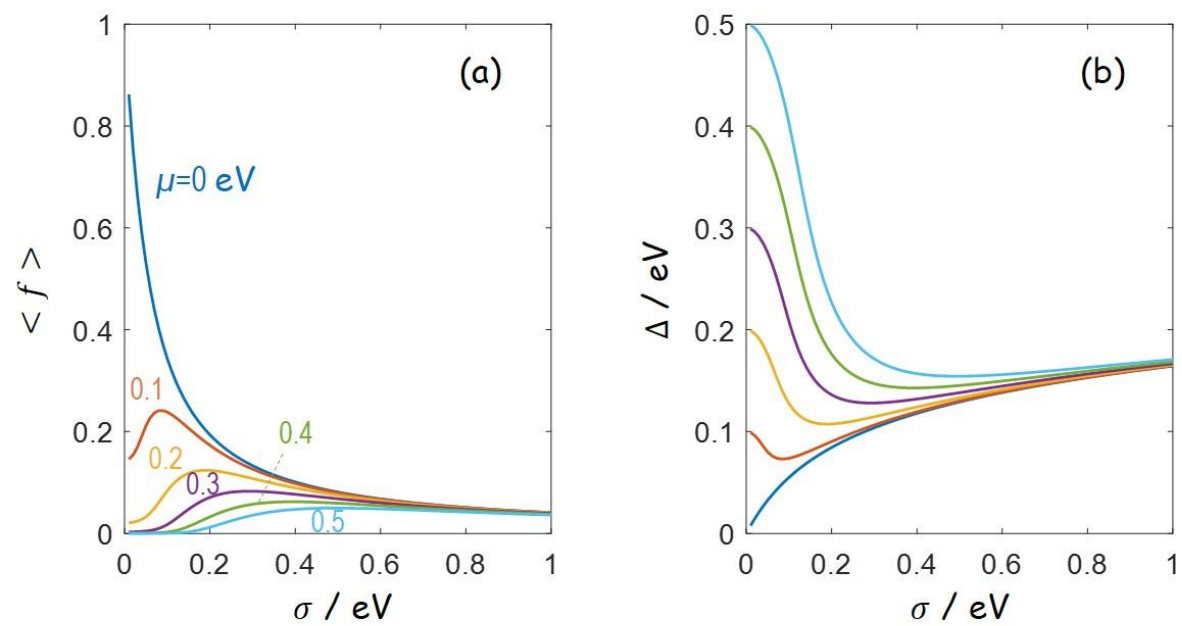

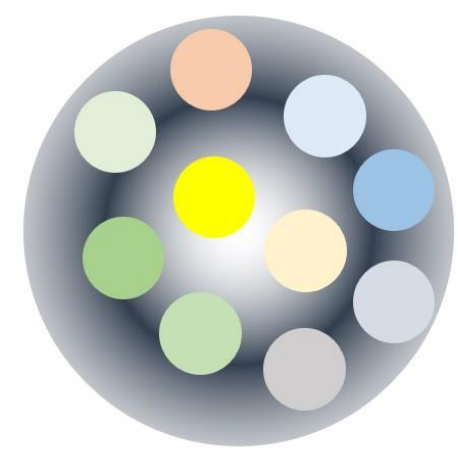

Nonhomogeneous

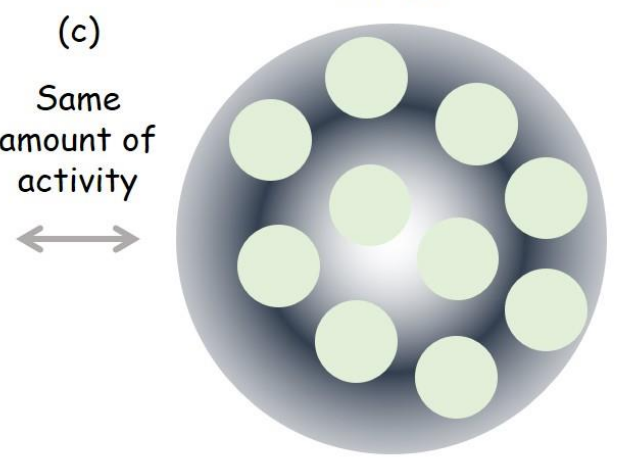

Homogeneous

Figure 1. (a) The overall catalytic activity $\langle f\rangle$ and (b) the positive-defined descriptor value of the homogenous counterpart $\Delta$ as a function of $\sigma$ for different values of $\mu$ (in steps of $0.1 \mathrm{eV}$ from 0 to $0.5 \mathrm{eV}$ ). (c) Schematic illustration of the homogeneous counterpart of a nonhomogeneous catalyst. Circles in different colors represent "active sites" with different binding energies. 


\section{Homogeneous counterpart}

A homogeneous counterpart is a homogeneous catalyst $(\sigma=0)$ that provides the same amount of catalytic activity as that of the nonhomogeneous catalyst, as illustrated in Figure $1(\mathrm{c})$. The descriptor value of this homogenous counterpart is denoted as $\Delta(\mathrm{eV})$ and adopts positive values only (note again that $\langle f\rangle$ is an even function of $x$ ). From the definition, we have $f(\Delta)=\langle f\rangle$, leading to

$$
\Delta(\mu, \sigma)=-\frac{\beta \sigma^{2}}{2}-\frac{1}{\beta} \ln \left(\frac{e^{\beta \mu} \operatorname{erfc}\left(\frac{\beta \sigma^{2}+\mu}{\sqrt{2} \sigma}\right)+e^{-\beta \mu} \operatorname{erfc}\left(\frac{\beta \sigma^{2}-\mu}{\sqrt{2} \sigma}\right)}{2}\right) .
$$

When $\mu \neq 0, e^{\beta|\mu|} \operatorname{erfc}\left(\frac{|\mu|+\beta \sigma^{2}}{\sqrt{2} \sigma}\right) \ll e^{-\beta|\mu|} \operatorname{erfc}\left(\frac{\beta \sigma^{2}-|\mu|}{\sqrt{2} \sigma}\right)$, and $\Delta$ is approximated as

$$
\Delta(\mu \neq 0) \approx|\mu|-\frac{\beta \sigma^{2}}{2}-\frac{1}{\beta} \ln \left(\frac{1}{2} \operatorname{erfc}\left(\frac{\beta \sigma^{2}-|\mu|}{\sqrt{2} \sigma}\right)\right)
$$

When $\mu=0$, Eq.(11) is reduced to

$$
\Delta(\mu=0)=-\frac{\beta \sigma^{2}}{2}-\frac{1}{\beta} \ln \left(\operatorname{erfc}\left(\frac{\beta \sigma}{\sqrt{2}}\right)\right)
$$

which, by using the approximation, $\operatorname{erfc}(\mathrm{x}) \approx \frac{1}{\sqrt{\pi}} \frac{e^{-x^{2}}}{x}$ for $x \gg \sqrt{2 / \pi}$, namely, $\sigma \gg \frac{2}{\beta} \sqrt{\frac{2}{\pi}}=$ $0.08 \mathrm{eV}$, could be further simplified to

$$
\Delta\left(\mu=0, \sigma \gg \frac{2}{\beta} \sqrt{\frac{2}{\pi}}\right)=\frac{1}{\beta} \ln \left(\sqrt{\frac{\pi}{2}} \beta \sigma\right) .
$$

Figure 1(b) visualizes how $\Delta$ varies as a function of $\mu$ and $\sigma$. At the vertical line $\sigma=0$, namely, for homogeneous electrocatalysts, we retrieve the expected result, $\Delta=\mu$. For a given $\mu, \Delta$ first decreases but then increases with raising $\sigma$, which is consistent with the nonmonotonic trend of $\langle f\rangle$ in Figure 1(a). All the curves converge to the same curve, $\Delta=\frac{1}{\beta} \ln \left(\sqrt{\frac{\pi}{2}} \beta \sigma\right)$, when $\sigma \gg$ 
$\max \left(\frac{2}{\beta} \sqrt{\frac{2}{\pi}}, \sqrt{\frac{|\mu|}{\beta}}\right)$, implying that the overall catalytic activity is nearly independent of the mean property when the standard deviation is sufficiently high. In other words, homogeneity control becomes more important than mean-property control in such situations.

\section{Site-site variation}

In addition to the overall catalytic activity, the activity variation between different active sites, termed site-site variation for short, is also of interest. An analytical expression for the site-site variation normalized with respect to the average activity, denoted by $\Sigma$, is obtained

$$
\Sigma=\sqrt{\frac{2 e^{(\beta \sigma)^{2}}\left[e^{-2 \beta \mu} \operatorname{erfc}\left(\frac{2 \beta \sigma^{2}-\mu}{\sqrt{2} \sigma}\right)+e^{2 \beta \mu} \operatorname{erfc}\left(\frac{2 \beta \sigma^{2}+\mu}{\sqrt{2} \sigma}\right)\right]}{\left[e^{-\beta \mu} \operatorname{erfc}\left(\frac{\beta \sigma^{2}-\mu}{\sqrt{2} \sigma}\right)+e^{\beta \mu} \operatorname{erfc}\left(\frac{\beta \sigma^{2}+\mu}{\sqrt{2} \sigma}\right)\right]^{2}}-1 .}
$$

Figure 2 shows $\Sigma$ as a function of $\sigma$ when $\mu$ varies from $0 \mathrm{eV}$ to $1 \mathrm{eV}$ (in steps of $0.1 \mathrm{eV}$ ). $\Sigma$ first increases exponentially, but then goes through a hump, and finally converges, regardless of $\mu$, to $\sigma^{0.5}$ with increasing $\sigma$. For a given $\sigma, \Sigma$ is greater with increasing $\mu$. Correspondingly, the activity variation between active sites is more pronounced when the mean binding energy deviates further from the volcano's apex. The nonmonotonic behavior of $\Sigma$ as a function of $\sigma$ for $\mu>0 \mathrm{eV}$ can be understood in the same way as in Figure 1 (a) and (b) corresponding to $\langle f\rangle$ and $\Delta$, respectively. 


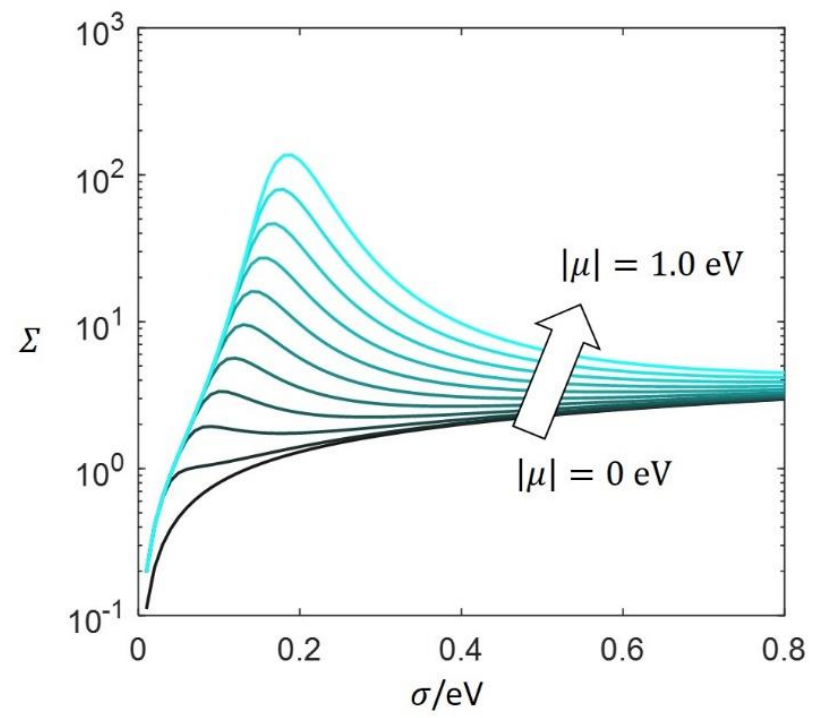

Figure 2. Normalized site-site variation $\Sigma$ as a function of $\sigma$ when $|\mu|$ increases from $0 \mathrm{eV}$ to $1 \mathrm{eV}$ in steps of $0.1 \mathrm{eV}$.

\section{Degradation of catalytic activity}

Figure 3 (a)-(c) shows the evolution of $p(x, t)$ for different initial conditions with a diffusion coefficient of $D=0.005\left(\mathrm{~m} \mathrm{eV}^{-2} \mathrm{~s}^{-1}\right)$ (an arbitrary value for the mere purpose of quantitative exposition; the results of Figure 3 (a)-(c) do not change if we use other values). For $\mu_{0}<0$ ( $\mu_{0}>$ $0)$, the mean of $p(x, t)$ is shifted to more negative (positive) values and $\sigma$ increases with aging. In case of $\mu_{0}=0$, however, the mean of $p(x, t)$ is kept virtually constant while $\sigma$ grows.

The degradation pattern, namely, the time dependency of $\langle f\rangle$, is dependent on the initial distribution. From asymptotic analysis of Eq.(4) with $\mu$ and $\sigma$ given in Eq.(9) and (10), respectively, we obtain that, if $\left|\mu_{0}\right|>\beta \sigma_{0}^{2}$, the degradation behavior initially follows

$$
\ln \langle f\rangle \propto-\beta\left[\gamma\left(\left|\mu_{0}\right|-\beta \sigma_{0}^{2}\right)+D\right] t
$$

and transitions to

$$
\ln \langle f\rangle \propto-\gamma t
$$

at a later stage; if $\left|\mu_{0}\right|<\beta \sigma_{0}^{2}$, only the latter pattern, $\ln \langle f\rangle \propto-\gamma t$, can be found. 
Figure 3 (d)-(e) shows the evolution of $\langle f\rangle$, normalized to its initial value, for different $\mu_{0}$ 's and different $D^{\prime}$ s, respectively. Given $\sigma_{0}=0.05 \mathrm{eV}$ and $D=\gamma \sigma_{0}^{2} / 2, \ln \langle f\rangle$ decreases with a slope of -1 as a function of $\gamma t$ for $\mu_{0}=0 \mathrm{eV}$, while it exhibits a two-stage degradation pattern for $\mu_{0}=$ $-0.2 \mathrm{eV}$, viz., $\left|\mu_{0}\right|>\beta \sigma_{0}^{2}$. The first stage becomes steeper when $D$ is greater, as shown in Figure $3(e)$.

The model results are tested using platinum-group metal-free (PGM-free) ORR catalysts in Figure 3 (f)-(g). The primary consideration of selecting experimental data is that they should be measured at a fixed potential, which is the case for PGM-free ORR catalysts.[46, 47] On the contrary, the durability of Pt-based ORR catalysts is usually evaluated by letting catalysts undergo potential cycling, according to standard protocols.[48]

Figure $3(\mathrm{f})-(\mathrm{g})$ show the activity degradation of two PGM-free ORR electrocatalysts belonging to the Fe/N/C catalyst family, including (f) the type A prepared by Shao et al.[46], and (g) the type B prepared by Yin and Zelenay.[47] Three type A electrocatalysts, prepared at three pyrolysis temperatures $\left(950,1050,1150{ }^{\circ} \mathrm{C}\right)$, are compared at $0.6 \mathrm{~V}_{\mathrm{RHE}}$ in a single $\mathrm{H}_{2} / \mathrm{O}_{2}$ cell. The type $\mathrm{B}$ electrocatalyst is evaluated at three different potentials $\left(0.84,0.70,0.40 \mathrm{~V}\right.$ RHE in a single $\mathrm{H}_{2} / \mathrm{O}_{2}$ cell at $80^{\circ} \mathrm{C}$.[47]

A two-stage decay pattern, consisting of an initial fast stage and a successive slow stage, is found for the two electrocatalysts, falling into the regime of $\left|\mu_{0}\right|>\beta \sigma_{0}^{2}$ in the present treatment. $\mu_{0}$ and $\sigma_{0}$ of these two electrocatalysts are not reported in the literature. The ill-defined nature and variety of active sites prevent us from a reliable determination of $\mu_{0}$ and $\sigma_{0}$ using electronic structure calculations. Thus, focus is put on the second stage, which follows a relation of $\ln \langle f\rangle \propto$ $-\gamma t$ according to the present treatment. Values for $\gamma$ are obtained unambiguously from the second stage, and are listed beside the corresponding curve in Figure 3. It is found that, $\gamma$ is lower, indicating retarded deactivation of active sites and slower degradation, when the pyrolysis temperature is higher and when the overpotential is lower; both trends are intuitively reasonable, lending credence to the present treatment. 

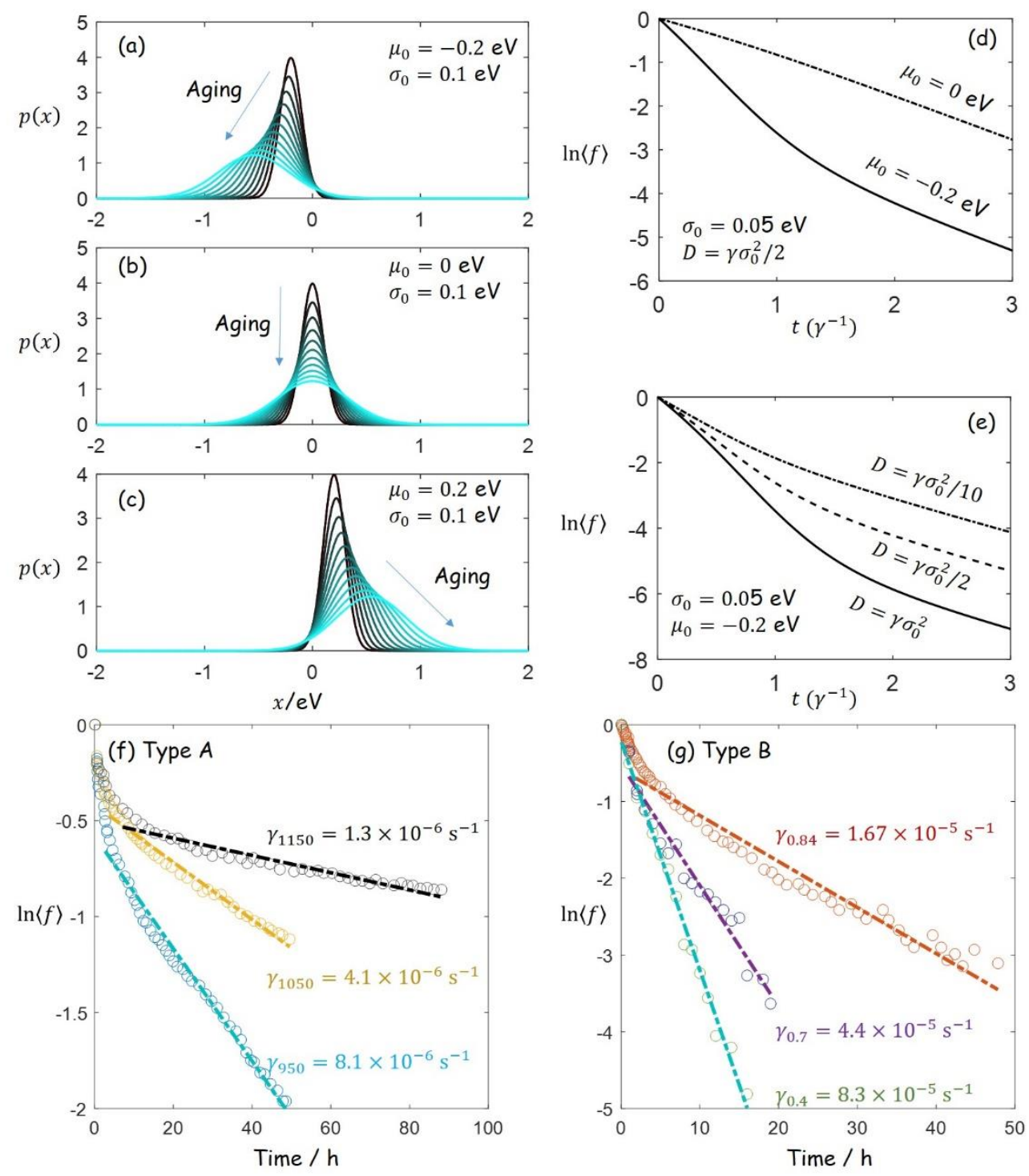

Figure 3. (a)-(c) Evolution of the probability distribution function from different initial conditions. The diffusion coefficient is $D=0.005\left(\mathrm{~m} \mathrm{eV}^{-2} \mathrm{~s}^{-1}\right)$, the time range is $\left(0, \gamma^{-1}\right)$, and the time step is $(10 \gamma)^{-1}$. Evolution of $\langle f\rangle$ normalized to its initial value for $(\mathrm{d})$ different initial mean values $\mu_{0}$ and (e) different diffusion coefficients $D$ with other conditions specified. (f) and (g) Fitting degradation behavior of platinum group metal-free (PGM-free) oxygen reduction electrocatalysts using the present model. Dashed lines represent model simulation, and symbols represent experimental data which have been normalized with respect to their initial values. (f) shows the results for NC-CITeTMPP electrocatalyst (type A) prepared by Shao et al.[46] Three pyrolysis temperatures $\left(950,1050,1150^{\circ} \mathrm{C}\right)$ are compared. The degradation tests were conducted 
using a single $\mathrm{H}_{2} / \mathrm{O}_{2}$ cell at $0.6 \mathrm{~V}_{\text {RHE. }}$ (g) shows the results for CM-PANI-Fe-C(Zn) electrocatalyst (type B) prepared by Yin and Zelenay.[47] The degradation tests were conducted using a single $\mathrm{H}_{2} / \mathrm{O}_{2}$ cell at $80^{\circ} \mathrm{C}$ at three different potentials $(0.84,0.70,0.40 \mathrm{VRH})$.

\section{Conclusion}

We developed a simple theoretical model with analytical solutions to describe statistical properties of electrocatalytic activity and durability. The model reveals that the overall catalytic activity of a nonhomogeneous catalyst is strongly dependent on the inhomogeneity. The highest overall catalytic activity is obtained when the standard deviation is commensurate with the mean value of the nonhomogeneous distribution. The site-site variation of activity is derived, which can be used as an analytical tool to interpret results obtained by electrocatalytic activity mapping methods. Degradation patterns are analytically related to the evolution kinetics of the distribution via the Fokker-Planck theory. Exponential decay of the overall catalytic activity is predicted and confirmed by experimental data of PGM-free ORR catalysts taken from the literature. The present model can be a valuable theoretical tool to characterize and further to design the heterogeneities of electrocatalysts.

\section{Acknowledgement}

This work is financially supported by the Alexander von Humboldt Foundation, and National Natural Science Foundation of China under the grant number of 21802170. The author is grateful to Dr. Kai Exner for insightful discussions.

\section{References and Notes}

[1] J.K. Nørskov, T. Bligaard, A. Logadottir, S. Bahn, L.B. Hansen, M. Bollinger, H. Bengaard, B. Hammer, Z. Sljivancanin, M. Mavrikakis, Y. Xu, S. Dahl, C.J.H. Jacobsen, Universality in Heterogeneous Catalysis, Journal of Catalysis, 209 (2002) 275-278.

[2] B. Hammer, J.K. Norskov, Why gold is the noblest of all the metals, Nature, 376 (1995) 238240. 
[3] A.J. Appleby, ELECTROCATALYSIS AND FUEL CELLS, Catalysis Reviews, 4 (1971) 221-244.

[4] S. Trasatti, Work function, electronegativity, and electrochemical behaviour of metals: III. Electrolytic hydrogen evolution in acid solutions, Journal of Electroanalytical Chemistry and Interfacial Electrochemistry, 39 (1972) 163-184.

[5] J. Rossmeisl, Z.W. Qu, H. Zhu, G.J. Kroes, J.K. Nørskov, Electrolysis of water on oxide surfaces, Journal of Electroanalytical Chemistry, 607 (2007) 83-89.

[6] F. Abild-Pedersen, J. Greeley, F. Studt, J. Rossmeisl, T.R. Munter, P.G. Moses, E. Skúlason, T. Bligaard, J.K. Nørskov, Scaling Properties of Adsorption Energies for Hydrogen-Containing Molecules on Transition-Metal Surfaces, Physical Review Letters, 99 (2007) 016105.

[7] A. Kulkarni, S. Siahrostami, A. Patel, J.K. Nørskov, Understanding Catalytic Activity Trends in the Oxygen Reduction Reaction, Chemical Reviews, 118 (2018) 2302-2312.

[8] V. Viswanathan, H.A. Hansen, J. Rossmeisl, J.K. Nørskov, Universality in Oxygen Reduction Electrocatalysis on Metal Surfaces, ACS Catalysis, 2 (2012) 1654-1660.

[9] K.S. Exner, Is Thermodynamics a Good Descriptor for the Activity? Re-Investigation of Sabatier's Principle by the Free Energy Diagram in Electrocatalysis, ACS Catalysis, 9 (2019) 53205329.

[10] J.K. Nørskov, T. Bligaard, B. Hvolbæk, F. Abild-Pedersen, I. Chorkendorff, C.H. Christensen, The nature of the active site in heterogeneous metal catalysis, Chemical Society Reviews, 37 (2008) 2163-2171.

[11] T. Bligaard, J.K. Nørskov, S. Dahl, J. Matthiesen, C.H. Christensen, J. Sehested, The BrønstedEvans-Polanyi relation and the volcano curve in heterogeneous catalysis, Journal of Catalysis, 224 (2004) 206-217.

[12] J.K. Nørskov, T. Bligaard, J. Rossmeisl, C.H. Christensen, Towards the computational design of solid catalysts, Nature Chemistry, 1 (2009) 37-46.

[13] I.E.L. Stephens, A.S. Bondarenko, U. Grønbjerg, J. Rossmeisl, I. Chorkendorff, Understanding the electrocatalysis of oxygen reduction on platinum and its alloys, Energy \& Environmental Science, 5 (2012) 6744-6762.

[14] E. Herrero, J.M. Feliu, Understanding formic acid oxidation mechanism on platinum single crystal electrodes, Current Opinion in Electrochemistry, 9 (2018) 145-150.

[15] M.T.M. Koper, Structure sensitivity and nanoscale effects in electrocatalysis, Nanoscale, 3 (2011) 2054-2073.

[16] N.M. Marković, P.N. Ross, Surface science studies of model fuel cell electrocatalysts, Surface Science Reports, 45 (2002) 117-229. 
[17] A.S. Bandarenka, M.T.M. Koper, Structural and electronic effects in heterogeneous electrocatalysis: Toward a rational design of electrocatalysts, Journal of Catalysis, 308 (2013) 1124.

[18] V. Čolić, A.S. Bandarenka, Pt Alloy Electrocatalysts for the Oxygen Reduction Reaction: From Model Surfaces to Nanostructured Systems, ACS Catalysis, 6 (2016) 5378-5385.

[19] S. Kolagatla, P. Subramanian, A. Schechter, Catalytic current mapping of oxygen reduction on isolated Pt particles by atomic force microscopy-scanning electrochemical microscopy, Applied Catalysis B: Environmental, 256 (2019) 117843.

[20] S. Kolagatla, P. Subramanian, A. Schechter, Simultaneous Mapping of Oxygen Reduction Activity and Hydrogen Peroxide Generation on Electrocatalytic Surfaces, ChemSusChem, 12 (2019) 2708-2714.

[21] A.J. Wain, Imaging size effects on the electrocatalytic activity of gold nanoparticles using scanning electrochemical microscopy, Electrochimica Acta, 92 (2013) 383-391.

[22] Y.-C. Weng, C.-T. Hsieh, Scanning electrochemical microscopy characterization of bimetallic Pt-M (M=Pd, Ru, Ir) catalysts for hydrogen oxidation, Electrochimica Acta, 56 (2011) 1932-1940.

[23] C.M. Sánchez-Sánchez, F.J. Vidal-Iglesias, J. Solla-Gullón, V. Montiel, A. Aldaz, J.M. Feliu, E. Herrero, Scanning electrochemical microscopy for studying electrocatalysis on shape-controlled gold nanoparticles and nanorods, Electrochimica Acta, 55 (2010) 8252-8257.

[24] Y. Wang, E. Gordon, H. Ren, Mapping the Potential of Zero Charge and Electrocatalytic Activity of Metal-Electrolyte Interface via a Grain-by-Grain Approach, Analytical Chemistry, 92 (2020) 2859-2865.

[25] F. Calle-Vallejo, M.D. Pohl, D. Reinisch, D. Loffreda, P. Sautet, A.S. Bandarenka, Why conclusions from platinum model surfaces do not necessarily lead to enhanced nanoparticle catalysts for the oxygen reduction reaction, Chemical Science, 8 (2017) 2283-2289.

[26] S.G. Rinaldo, J. Stumper, M. Eikerling, Physical Theory of Platinum Nanoparticle Dissolution in Polymer Electrolyte Fuel Cells, The Journal of Physical Chemistry C, 114 (2010) 5773-5785.

[27] S.G. Rinaldo, P. Urchaga, J. Hu, W. Lee, J. Stumper, C. Rice, M. Eikerling, Theoretical analysis of electrochemical surface-area loss in supported nanoparticle catalysts, Physical Chemistry Chemical Physics, 16 (2014) 26876-26886.

[28] S.G. Rinaldo, W. Lee, J.r. Stumper, M. Eikerling, Model- and Theory-Based Evaluation of Pt Dissolution for Supported Pt Nanoparticle Distributions under Potential Cycling, Electrochemical and Solid-State Letters, 14 (2011) B47.

[29] B. Hammer, J.K. Nørskov, Electronic factors determining the reactivity of metal surfaces, Surface Science, 343 (1995) 211-220. 
[30] J.K. Nørskov, F. Studt, F. Abild-Pedersen, T. Bligaard, Poisoning and Promotion of Catalysts, in: Fundamental Concepts in Heterogeneous Catalysis, 2014, pp. 150-154.

[31] J. Huang, J. Zhang, M. Eikerling, Unifying theoretical framework for deciphering the oxygen reduction reaction on platinum, Physical Chemistry Chemical Physics, 20 (2018) 11776-11786.

[32] L. Zhang, J. Huang, Understanding Surface Charge Effects in Electrocatalysis. Part I: Peroxodisulfate Reduction at Pt(111), The Journal of Physical Chemistry C, 124 (2020) 1695116960.

[33] X. Zhu, J. Huang, Modeling Electrocatalytic Oxidation of Formic Acid at Platinum, Journal of The Electrochemical Society, 167 (2019) 013515.

[34] M.K. Sabbe, M.-F. Reyniers, K. Reuter, First-principles kinetic modeling in heterogeneous catalysis: an industrial perspective on best-practice, gaps and needs, Catalysis Science \& Technology, 2 (2012) 2010-2024.

[35] H.A. Hansen, V. Viswanathan, J.K. Nørskov, Unifying Kinetic and Thermodynamic Analysis of 2 e- and 4 e- Reduction of Oxygen on Metal Surfaces, The Journal of Physical Chemistry C, 118 (2014) 6706-6718.

[36] C.F. Dickens, C. Kirk, J.K. Nørskov, Insights into the Electrochemical Oxygen Evolution Reaction with ab Initio Calculations and Microkinetic Modeling: Beyond the Limiting Potential Volcano, The Journal of Physical Chemistry C, 123 (2019) 18960-18977.

[37] M.T.M. Koper, Analysis of electrocatalytic reaction schemes: distinction between ratedetermining and potential-determining steps, Journal of Solid State Electrochemistry, 17 (2013) 339-344.

[38] U. Martinez, S. Komini Babu, E.F. Holby, P. Zelenay, Durability challenges and perspective in the development of PGM-free electrocatalysts for the oxygen reduction reaction, Current Opinion in Electrochemistry, 9 (2018) 224-232.

[39] T. Asset, P. Atanassov, Iron-Nitrogen-Carbon Catalysts for Proton Exchange Membrane Fuel Cells, Joule, 4 (2020) 33-44.

[40] L. Du, V. Prabhakaran, X. Xie, S. Park, Y. Wang, Y. Shao, Low-PGM and PGM-Free Catalysts for Proton Exchange Membrane Fuel Cells: Stability Challenges and Material Solutions, Advanced Materials, n/a (2020) 1908232.

[41] A. Nitzan, Chemical dynamics in condensed phases: relaxation, transfer and reactions in condensed molecular systems, Oxford university press, 2006.

[42] Y. Zhang, J. Zhang, J. Huang, Potential-Dependent Volcano Plot for Oxygen Reduction: Mathematical Origin and Implications for Catalyst Design, The Journal of Physical Chemistry Letters, 10 (2019) 7037-7043. 
[43] K.S. Exner, Activity-Stability Volcano Plots for Material Optimization in Electrocatalysis, ChemCatChem, 11 (2019) 3234-3241.

[44] J. Chen, Y. Chen, P. Li, Z. Wen, S. Chen, Energetic Span as a Rate-Determining Term for Electrocatalytic Volcanos, ACS Catalysis, 8 (2018) 10590-10598.

[45] H. Ooka, R. Nakamura, Shift of the Optimum Binding Energy at Higher Rates of Catalysis, The Journal of Physical Chemistry Letters, 10 (2019) 6706-6713.

[46] M. Shao, Q. Chang, J.-P. Dodelet, R. Chenitz, Recent Advances in Electrocatalysts for Oxygen Reduction Reaction, Chemical Reviews, 116 (2016) 3594-3657.

[47] X. Yin, P. Zelenay, (Invited)Kinetic Models for the Degradation Mechanisms of PGM-Free ORR Catalysts, ECS Transactions, 85 (2018) 1239-1250.

[48] H. Chen, Z. Song, X. Zhao, T. Zhang, P. Pei, C. Liang, A review of durability test protocols of the proton exchange membrane fuel cells for vehicle, Applied Energy, 224 (2018) 289-299. 